\title{
Eukaryotic DNA damage tolerance and translesion synthesis through covalent modifications of PCNA
}

\author{
Parker L Andersen ${ }^{1}$, Fang $\mathrm{Xu}^{2}$, Wei Xiao ${ }^{1}$ \\ ${ }^{I}$ Department of Microbiology and Immunology, University of Saskatchewan, Saskatoon, SK, Canada S7N 5E5, USA; ${ }^{2}$ Department \\ of Biology, Ningxia Medical College, Yinchuan, Ningxia 750004, China
}

In addition to well-defined DNA repair pathways, all living organisms have evolved mechanisms to avoid cell death caused by replication fork collapse at a site where replication is blocked due to disruptive covalent modifications of DNA. The term DNA damage tolerance (DDT) has been employed loosely to include a collection of mechanisms by which cells survive replication-blocking lesions with or without associated genomic instability. Recent genetic analyses indicate that DDT in eukaryotes, from yeast to human, consists of two parallel pathways with one being error-free and another highly mutagenic. Interestingly, in budding yeast, these two pathways are mediated by sequential modifications of the proliferating cell nuclear antigen (PCNA) by two ubiquitination complexes Rad6-Rad18 and Mms2-Ubc13-Rad5. Damage-induced monoubiquitination of PCNA by Rad6-Rad18 promotes translesion synthesis (TLS) with increased mutagenesis, while subsequent polyubiquitination of PCNA at the same K164 residue by Mms2-Ubc13-Rad5 promotes error-free lesion bypass. Data obtained from recent studies suggest that the above mechanisms are conserved in higher eukaryotes. In particular, mammals contain multiple specialized TLS polymerases. Defects in one of the TLS polymerases have been linked to genomic instability and cancer.

Keywords: DNA damage tolerance, translesion synthesis, Y-family polymerase, ubiquitination, sumoylation, PCNA Cell Research (2008) 18:162-173. doi: 10.1038/cr.2007.114; published online 24 December 2007

\section{DNA damage tolerance}

In the presence of spontaneous or carcinogen-induced DNA damage, living cells have to maintain and complete DNA synthesis or risk replication fork collapse. Since the process of DNA licensing is to ensure the genome is duplicated once and only once during each cell cycle, stalled or collapsed replication forks may not be able to restart, which often results in double-strand breaks (DSBs) and causes compromised genome integrity or cell death. In addition to highly conserved DNA repair pathways, all living organisms have evolved schemes to ensure continuation of DNA synthesis in the presence of damage. These schemes were originally termed DNA postreplication repair (PRR) due to observations of transient shortened nascent DNA structures following $\mathrm{S}$ phase in response to DNA damage. In bacteria and unicellular yeast, these shortened DNA

Correspondence: Wei Xiao

Tel: +1 306966 4308; Fax: +1 3069664311

E-mail:wei.xiao@usask.ca segments can be measured by an alkaline sedimentation assay [1] or directly observed in electron micrographs [2]. In wild-type cells, these truncated DNA segments were restored to full length following a short recovery time. One typical experiment [1] involved the restoration of the nascent strand following UV exposure in nucleotide excision repair (NER)-deficient cells and was originally assumed to represent a mechanism of DNA repair. However, further investigation revealed that, although the nascent fragments were re-annealed, the original UV-induced pyrimidine dimers, which were responsible for the generation of single-strand gaps, often persisted in the genome [3, 4]. It was argued that the replication-blocking lesion was not necessarily corrected, but rather transiently bypassed and carried over to the next generation. Perhaps it is more beneficial for the organism to tolerate DNA damage rather than to allow replication fork collapse. Since, unlike other DNA repair mechanisms, this pathway does not actually remove damage-induced lesions, we propose to use the term DNA damage tolerance (DDT) to describe this general phenomenon. In both prokaryotes and eukaryotes, DDT is accomplished by alternative mechanisms with rather 
different biological consequences and these mechanisms appear to be evolutionarily conserved. In this review, we will focus on recent advances pertaining to cohesive DDT mechanisms mediated by covalent modifications of proliferating cell nuclear antigen (PCNA) in eukaryotes. Readers are encouraged to refer to recent review articles [5-7] for this general field.

\section{DDT in prokaryotes}

Early experiments using an NER mutant in Escherichia coli demonstrated DNA gap formation in the nascent strand opposite UV-induced thymidine dimers [8], suggesting replication restart downstream of the blocking lesion. Using an alkaline sedimentation assay, it was demonstrated that these gaps were transient [9] and the persistence of lesions in subsequent generations suggested the existence of a DDT mechanism in E. coli [4]. This damage tolerance mechanism is thought to be dependent upon the bacterial SOS response, in which single-strand DNA (ssDNA) is recognized and bound by the RecA protein [10], which promotes two parallel pathways. Activated RecA (RecA*) induces the self-cleavage of the LexA repressor that in turn allows the transcription of various genes required for DNA repair and cell survival [11]. SOS induces the expression of both $\operatorname{Din} B$ and the $u m u D C$ operon encoding two polymerases, PolIV and PolV, respectively, for translesion synthesis (TLS). In addition, RecA* stimulates the cleavage of the regulatory subunit UmuD to form a fully functional PolV (UmuD' ${ }_{2}$-UmuC) $[12,13]$, and the RecA-ssDNA filaments are required for both homologous recombination and TLS [14]. This ssDNA-binding activity of RecA has been suggested to induce fork regression forming a chicken-foot structure, or to act as a primer for TLS allowing DDT and replication restart [15], as illustrated in Figure 1.

\section{Ubiquitination and sumoylation}

It is apparent that, in bacterial cells, RecA plays central and multiple roles in DDT. In contrast, the eukaryotic RecA sequence homolog Rad51 and its various paralogs in higher eukaryotes inherit the ssDNA filament formation and homologous recombination activity but do not confer regulatory functions, nor are they required for DDT. Recent studies show that eukaryotes employ a completely different mechanism to coordinate DDT, namely covalent modifications of a substrate by ubiquitin $(\mathrm{Ub})$ and small Ub-like modifier (SUMO), processes not found in bacteria. For this reason, it is important to briefly review the process of ubiquitination. $\mathrm{Ub}$ is a highly conserved 76 amino-acid protein that can be specifically attached to the $\varepsilon$-amino group of a Lys residue on a target protein in a three-step manner.
Firstly, in an ATP-dependent manner, Ub is linked by a thioester bond to the Ub-activating enzyme (Uba or E1). $\mathrm{Ub}$ is then transferred to an active site cysteine residue of a Ub-conjugating enzyme (Ubc or E2), which often operates along with a Ub ligase (E3) for target specificity. Ub is then transferred to the target protein, forming an isopeptide bond between the $\mathrm{C}$-terminus of $\mathrm{Ub}$ and the $\varepsilon$-amino group of a Lys residue. Covalently bound $\mathrm{Ub}$ is often further modified by sequential addition of $\mathrm{Ub}$ molecules to already bound $\mathrm{Ub}$ peptides to produce poly-Ub chains. The most characterized function of $\mathrm{Ub}$ modification is proteasome-dependent protein degradation of substrates modified with Ub chains joined sequentially at the Lys48 residue of the previous Ub [16]. However, it becomes clear that alternative Ub modifications influence diverse activities [17]. Such specifications may involve distinct linkages, such as Lys63-linked poly-Ub chains, or simple mono-Ub additions [18]. It is generally believed that these alternative Ub modifications differ from Lys48-linked Ub chains in that they often regulate the target protein activity instead of its degradation.

Eukaryotic cells also contain several classes of Ub-like molecules that adopt a Ub-like fold with conserved positioning of C-terminal residues for isopeptide bond formation and target protein modification [19, 20]. Each class employs a specific E2-E3 complex for target conjugation. SUMO is probably the most extensively characterized Ub-like molecule and its conjugation (sumoylation) often alters the target protein activity [21, 22].

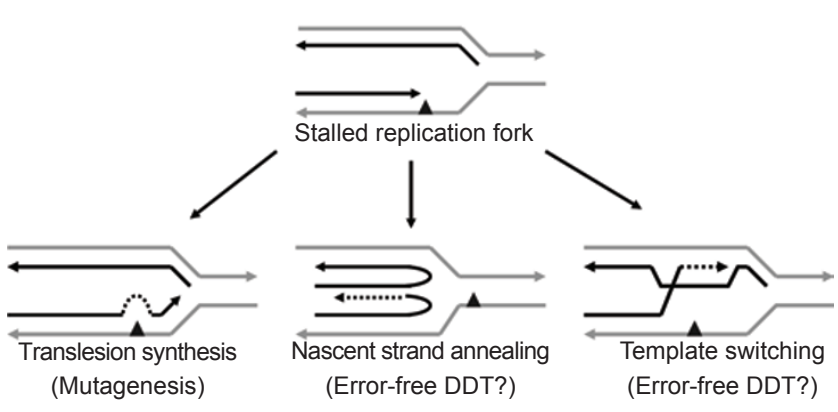

Figure 1 Possible mechanisms of DNA damage tolerance through replicative bypass of stalled replication forks. A triangle on the bottom template strand represents a replication block that stalls the replication machinery. Most organisms possess specialized polymerases capable of translesion synthesis across the block, often associated with an increased mutation rate. An error-free DDT utilizes newly synthesized sister chromatid as a template; however, it is subject to debate whether it is achieved through fork regression followed by nascent strand annealing and synthesis (the chicken-foot model), or through sister chromatid invasion followed by synthesis and Holliday junction resolution (the template switching model). 


\section{DDT in Saccharomyces cerevisiae}

Eukaryote DDT has been most extensively characterized in the budding yeast $S$. cerevisiae. Initially an alkaline sedimentation assay was employed to demonstrate the existence of DDT activity in yeast, and genetic analyses indicate that, in the absence of NER ( $\mathrm{radl}$ ), UV-induced ssDNA gaps cannot be filled in the rad6 or rad18 mutants [23]. RAD6 was the founding member of the PRR and mutagenesis pathway, one of the three major radiation repair pathways. However, historically, $R A D$ genes that do not belong to either of the well-defined RAD3 (NER) or RAD52 (homologous recombination repair (HRR)) group were assigned to the RAD6 pathway [24, 25]. The rad6 mutant exhibits a mutator and slow growth phenotype, is defective in UVinduced mutagenesis, and becomes extremely sensitive to killing by UV and a variety of DNA damaging agents [25, 26]. The rad6 diploid is also defective in sporulation [27]. $R A D 6$ was found to encode an E2 enzyme (Ubc2) [28] and its $\mathrm{Ub}$ conjugation activity is absolutely required for all of its functions [29]. The C-terminal polyacidic tail of Rad6 is required for its ability to polyubiquitinate histone $\mathrm{H} 2 \mathrm{~B}$ in vitro [30] and in vivo [31]. However, deletion of the entire Rad6 C-terminal tail has little effect on its role in DNA repair and UV-induced mutagenesis, and affects only sporulation [32]. The N-terminal 15 amino-acid sequence is nearly identical among all Rad6 homologs [33-36]; deletion of the first 9 amino acids from Rad6 $\left(\operatorname{rad}_{\Delta 1-9}\right)$ abolishes sporulation, reduces cell survival after UV treatment, but surprisingly increases spontaneous and UV-induced mutagenesis [37]. Furthermore, the N-terminus of Rad6 is also required for $\mathrm{N}$-end rule protein degradation $[29,37,38]$ : while the full-length Rad6 interacts with the E3 protein Ubr1, the Rad6 $_{\Delta 1-9}$ protein is unable to form a complex with Ubr1 [37]. Rad6 is known to form a stable complex with Rad18 [39], and this complex displays Ub conjugation (from Rad6), ssDNA-binding and ATPase (from Rad18) activities [40]. However, Rad18 had not been defined as an E3 until the RING finger motif was discovered [41, 42] and found in Rad18, and the physical interaction of Rad18 with the substrate Pol30 (PCNA) was demonstrated [43]. Like rad6, the rad18 mutant is extremely sensitive to killing by UV and a variety of DNA damaging agents, and displays a mutator phenotype [44]; however, unlike rad6, rad18 displays a signature spontaneous GC-to-TA mutation increase [45] and does not display slow growth and sporulation defects [26]. Hence, Rad6 appears to be a multi-functional E2 with different partners, and its DDT activity is exclusively achieved through interaction with $\operatorname{Rad} 18$ [5].

Further genetic analysis has demonstrated that the RAD6 pathway can be divided into two parallel pathways, one be- ing error-prone and another error-free. The error-prone or mutagenesis pathway was first discovered through genetic screens of rev mutants incapable of reverting the arg 4-17 and lys $1-1$ alleles in response to UV irradiation [46, 47]. $R E V 1$ was cloned and found to encode a $112-\mathrm{kDa}$ protein [48] with deoxycytidyl transferase activity [49], whereas REV 3 and REV7 encode two subunits of a non-essential DNA polymerase, Pol $\zeta$, capable of bypassing thymine dimers more efficiently than Pol $\alpha$ [50]. The rev mutants exhibit moderate sensitivity to a variety of DNA damaging agents, but with strongly compromised mutability [51]. Thus, the yeast mutagenesis pathway relies on a non-essential DNA polymerase to bypass DNA replication blocks, or TLS, at the cost of increased mutagenesis.

The rad 6 and rad 18 mutations are epistatic to rev mutations; however, it is apparent that TLS is not the only pathway operated by RAD6-RAD18, since the rad6 or rad18 mutants are much more sensitive to DNA damaging agents than the rev mutants [26]. An error-free branch within the RAD6 pathway had been proposed but not convincingly demonstrated until the identification and functional characterization of MMS2 [52]. The mms 2 mutant is moderately sensitive to a broad range of DNA damaging agents, and epistasis analysis places $M M S 2$ within the RAD6 pathway. However, unlike rev3, the mms 2 mutant displays a massively increased spontaneous mutation rate and this increase is dependent on $R E V$ functions. Furthermore, the $m m s 2$ and rev3 mutations are synergistic with respect to DNA damage sensitivity and the double mutant is comparable to that of the rad18 single mutant [52, 53]. Based on these analyses, a model was proposed in which the RAD6 pathway is composed of two independent subpathways: one is mediated by TLS that requires $R E V 1,3$ and 7 , whereas the other is mediated by error-free PRR that requires $M M S 2$ [52]. MMS2 encodes a protein homologous to Ubc but lacking the active Cys residue [52]. It turns out that Mms2 forms a stable complex with a true Ubc, Ubc13, and that the Mms2-Ubc13 complex specifically catalyzes the formation of Lys63-linked Ub chains [54]. Indeed, the $u b c 13$ mutant displays phenotypes indistinguishable from those of the mms 2 mutant [55]. The cognate E3 for Mms2-Ubc13 turns out to be Rad5, another RING-finger protein that interacts with both $\mathrm{Ubc} 13$ and $\operatorname{Rad} 18$ [56]. RAD5 encodes a protein with DNA helicase and zinc-binding domains [57] and DNA-dependent ATPase activity [58]. Hence, at least two E2-E3 complexes, namely Rad6-Rad18 and Mms2Ubc13-Rad5, are required for DDT in yeast. In addition, $R A D 5$ has been reported to promote instability of simple repetitive sequences [57] and to inhibit non-homologous end-joining of DSBs [59]. Indeed, Rad5 is involved in double-strand break repair independent of its ubiquitination activity [60]. 


\section{Sequential modifications of PCNA}

PCNA (encoded by POL30 in budding yeast) forms a homotrimer which circles the DNA and operates as a scaffold, often termed a processivity factor, to assemble a multitude of proteins required for DNA unwinding and synthesis, cell cycle progression and chromatin structure maintenance [7]. The involvement of this DNA-polymerase sliding clamp in DDT was first suggested by the isolation and characterization of the pol30-46 allele [61]. pol30-46 is epistatic to rad6 and rad18, but synergistic with rev3. The pol30-46 mutant is normal in UV-induced mutagenesis and DNA synthesis but displays significantly reduced PRR activity as judged by the alkaline sedimentation assay [61].

PCNA can be either ubiquitinated or sumoylated in budding yeast [43]. In response to DNA damage, PCNA is modified by a single $\mathrm{Ub}$ on the Lys 164 residue and this process is dependent on the Rad6-Rad18 complex [43]. $\mathrm{Ub}$ modification appears to be limited to the PCNA that has been loaded onto DNA by replication factor C [62], suggesting that PCNA is monoubiquitinated only at stalled replication forks. In wild-type cells, polyubiquitinated PCNA was also observed upon DNA damage, and this modification is also at the Lys164 residue, linked through the Lys63 Ub chain, and requires functional MMS2, UBC13 and RAD5 [43]. Hence, it is conceivable that the two ubiquitination complexes Rad6-Rad18 and Mms2-Ubc13-Rad5 sequentially ubiquitinate PCNA. Interestingly, the identical residue can also be targeted for sumoylation; the fraction of sumoylated PCNA increases during S phase as well as during extensive DNA damage, and this process requires yet another E2-E3 complex Ubc9-Siz1 [43, 63]. It is noted that PCNA can also be sumoylated at the Lys 127 residue [43], and this specific modification does not appear to affect the DDT activity, but is required for the establishment of sister chromatid cohesion during S phase [64].

The discovery of PCNA covalent modifications imposes several functional implications. Firstly, it predicts that the pol30-164R mutation is epistatic to all DDT pathway mutations. Indeed, Pol30-164R cannot be ubiquitinated and the pol30-164R mutation suppresses the severe sensitivity of rad6 and rad18 mutations [43]. Secondly, it predicts that monoubiquitinated PCNA promotes TLS, which was subsequently demonstrated [63]. Thirdly, the above model suggests that polyubiquitinated PCNA promotes error-free DDT. To date, this prediction has not been explored. Finally, it indicates that the Pol30-K164 sumoylation plays a role in the regulation of DDT. Interestingly, the pol30-164R mutant is less sensitive to DNA damage than $\mathrm{rad} 6$, $\mathrm{rad} 18$ or the mms 2 rev 3 double mutant, suggesting that the Pol30K164 sumoylation sensitizes cells to DNA damage. This model is further strengthened by analyzing the effects of siz1 mutation that specifically affects sumoylation but not ubiquitination (UBC9 is an essential gene), and is reminiscent of the srs2 (suppression of rad six) mutation that was initially isolated by its ability to suppress the severe damage sensitivity of rad6 mutants [65]. Srs2 possesses a $3^{\prime}$ to $5^{\prime}$ DNA helicase activity $[66,67]$ that is crucial for recombination [67] and suppression of DDT defects [68, 69]. Genetic data indicate that Srs 2 negatively regulates recombination $[70,71]$ possibly by reversal of intermediate recombination structures [72-75]. Indeed, the DNA strand exchange mediated by Rad51 is inhibited by Srs 2 through disruption of the Rad51-ssDNA filaments [76, 77], and it turns out that sumoylated PCNA has increased affinity for Srs2 [78, 79] and represses the Rad52-dependent recombination pathway [80]. These observations collectively support the hypothesis that Srs2 serves as a molecular switch between homologous recombination and DDT [6], and further confirm that the sensor for this switch is the state of PCNA modification.

The current model of yeast DDT through covalent modifications of PCNA is depicted in Figure 2.

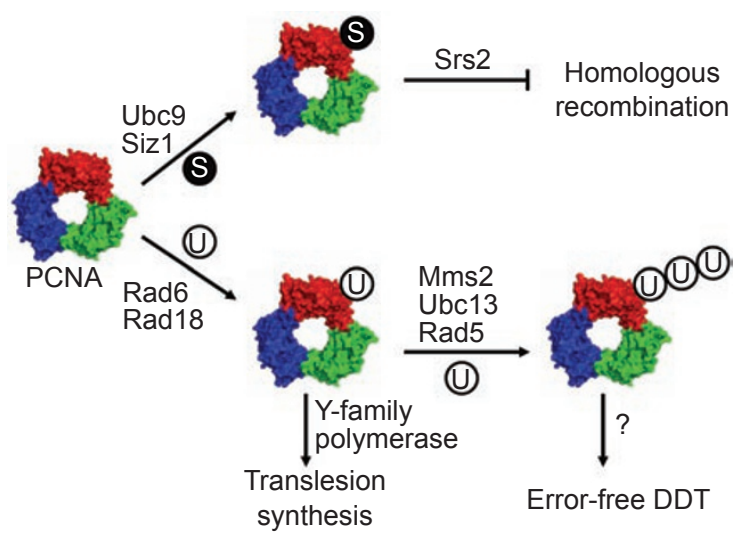

Figure 2 DNA damage tolerance through covalent modifications of PCNA, a model well established in the lower eukaryote $S$. cerevisiae and presumably conserved in higher eukaryotes. The PCNA homotrimer is illustrated in different colors and can be modified by either SUMO (S) or Ub (U) at the same Lys164 residue [43]. In the latter case, monoubiquitinated PCNA can be further polyubiquitinated by the E2-E3 complex Mms2-Ubc13-Rad5 to form Lys63-linked chains. Sumoylated PCNA is known to recruit the Srs2 helicase to disrupt the Rad51-ssDNA filament and prevent inappropriate homologous recombination; monoubiquitinated PCNA enhances affinity for Y-family polymerases to facilitate TLS, whereas polyubiquitinated PCNA is thought to promote error-free DDT, although it is unclear at present how this is achieved. Note that it is subject to debate whether just one or all three subunits of PCNA are modified to execute its functions. Modification of only one subunit is shown for simplicity. 


\section{Y family DNA polymerases}

Error-prone TLS can occur by the regular replicative polymerases or specialized, error-prone polymerases. Replicative polymerases include PolI, PolII and PolIII in prokaryotes, $\operatorname{Pol}(\alpha), \operatorname{Pol} 2(\varepsilon)$ and $\operatorname{Pol} 3(\delta)$ in yeast, and Pol $\alpha$, Pol $\varepsilon$ and Pol $\delta$ in higher eukaryotes. Errors can arise by simple incorrect base-pairing and/or lack of proofreading. Frameshift mutations often occur in regions of repeated nucleotide sequences likely from slippage of the template strand. In addition, certain nucleotide repeats can readily form secondary structures that become recombination hotspots and fragile sites in the DNA, among which triplet repeats can also provide sources of extensive amino-acid expansion in the coding region [81]. Furthermore, replicative polymerases may be required for extension from nucleotide insertion by a low-fidelity polymerase, thus stabilizing a potential mutation.

Essentially all TLS polymerases except one (i.e., Pol $\zeta$ ) are Y-family polymerases that lack a $3^{\prime}-5^{\prime}$ proofreading exonuclease activity and contain relatively non-restrictive active sites compared with the replicative polymerases [82] (Figure 3). Surprisingly, although members of this family of proteins have been studied for many years and implicated in mutagenesis or TLS, it was only at the end of the last century when they were reported as a novel class of DNA polymerases. This review is not intended to cover a comprehensive analysis of each Y-family polymerase. Readers are referred to excellent review articles that cover this topic [82-86] and an accompanying review article (McCulloch and Kunkel in this issue) discussing the fidelity of eukaryotic DNA polymerases. Rather, this section aims to provide an in-depth analysis of current literature on how monoubiquitinated PCNA leads to TLS. In addition, an accompanying review article (Gan et al. in this issue) provides an in-depth analysis of eukaryotic Pol $\zeta$.

E. coli contains two Y-family polymerases, PolIV and PolV; both are DNA damage inducible and belong to the SOS regulon. PolIV has an extremely low affinity for the naked primer-template substrate and heavily relies on the $\beta$ clamp (a bacterial functional homolog of PCNA) to load onto DNA $[87,88]$. In vitro studies indicate that PolIV and its archeal homolog Dpo4 are relatively faithful polymerases at the incorporation step and the low fidelity primarily results from poor discrimination between correct and incorrect incoming nucleotides at the binding stage and the capacity to elongate mismatched primer template, which results in -1 frameshift mutations $[89,90]$. Hence, PolIV promotes mutagenesis through three distinct mechanisms: replication error, TLS and incorporation of base analogs.

PolV plays a critical role in the most characterized damage-induced mutagenesis pathway. Like PolIV, DNA synthesis by PolV is strictly distributive, requires additional cofactors such as RecA, SSB and the $\beta$ clamp, and can efficiently bypass essentially all lesions tested to date [91]. PolV accounts for the vast majority of UV-induced mutagenesis in E. coli [91]. Due to its notorious substrate plasticity, PolV must be placed under strict regulation; indeed PolV activity is controlled at both transcriptional and post-translational levels [81].

Budding yeast also contains two Y-family polymerases. Rev1, the first characterized eukaryotic Y-family member, is a deoxycytidyl transferase that inserts a dCMP efficiently opposite a template abasic site and is probably responsible

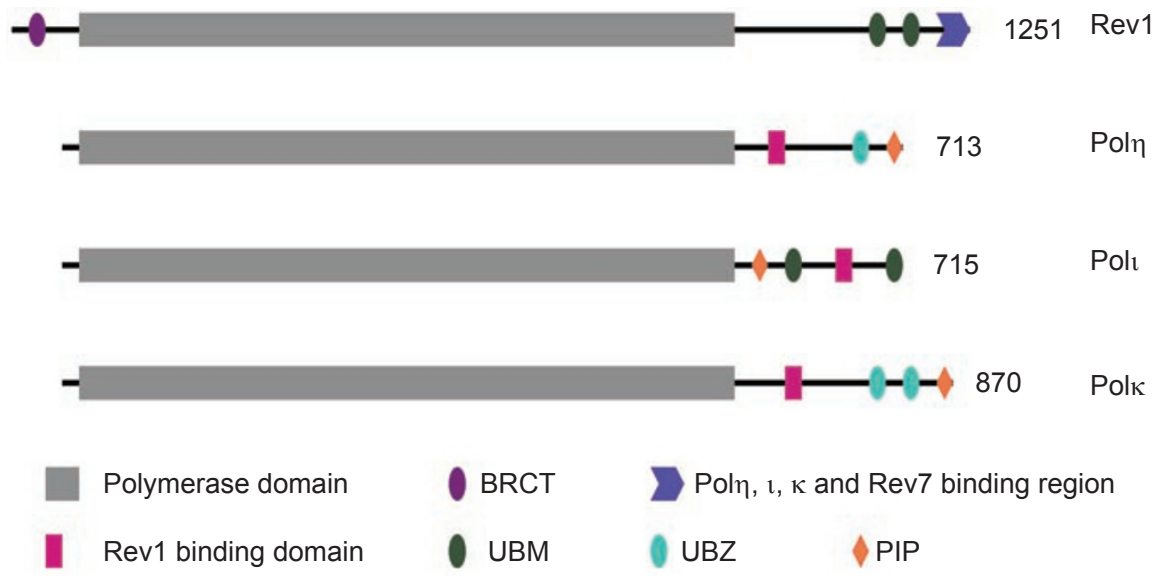

Figure 3 Functional domains of Y-family polymerases. Only four well-defined human Y-family polymerases are illustrated with the relative position of their functional domains indicated. Numbers indicate total amino acids of each protein. Note that the polymerase domain can be divided into subdomains based on structural analysis, which makes each of them specialized for lesion recognition and bypass. Other domains are considered to perform regulatory roles. Figure adapted from [82]. Abbreviations used: BRCT, BRAC1 C-terminal domain; UBM, Ub-binding motifs; UBZ, Ub-binding zinc fingers; PIP, PCNA interacting peptide. 
for $60-85 \%$ of the bypass events at a specific abasic site in vivo $[49,92]$. It can also insert dCMP across template $\mathrm{G}$ or A, albeit to a lesser extent [49]. Structural analysis indicates that Rev1 does not facilitate base pairing between the template $\mathrm{G}$ and the incoming dCTP. Instead, the $\mathrm{G}$ is evicted from the DNA helix and the dCTP pairs with a protein "template" Arg residue [93], which ensures base selection in a DNA template-independent manner. The yeast rev1 mutant displays a complete loss of mutagenesis activity comparable to that of rev3, which cannot be explained by its dCMP transferase activity. Indeed, analysis of site-specific mutations confirms that the Rev1 enzymatic activity is not essential for TLS, but its BRCA1 C-terminal (BRCT) domain [94, 95] and/or a polymerase-associated domain (PAD) [96] are required for protein interactions. The C-terminal 100 amino acids of human Rev1 are sufficient to interact with all other TLS polymerases [97] (Figure 3), implying a scaffold role of Rev1 in TLS. The Rev1 structure and functions appear to be highly conserved in higher eukaryotes. Experimental reduction of REVI expression in cultured human cells results in a decrease in UV-induced mutagenesis [98].

Pol $\eta$ in yeast is encoded by $R A D 30$, whose inactivation [99] or mutations in the corresponding mammalian xeroderma pigmentosum variant $(X P V)$ gene $[100,101]$ lead to an increased susceptibility to UV-induced DNA damage. Pol $\eta$ is able to correctly incorporate AA opposite cis-syn thymine-thymine dimers [102] with kinetics comparable to that of the opposite undamaged template [103]. This insertion fidelity is thought to be achieved through an induced-fit mechanism similar to replicative polymerases [104]. However, for other types of lesions including those induced by UV, such as cyclobutane pyrimidine dimers and TT (6-4) photoproducts, Pol $\eta$ has reduced affinity, poor incorporation rates or low fidelity [105]. Hence, Pol $\eta$ appears to be highly specialized and the only known "error-free" Y-family polymerase when bypassing thymine dimers.

Mammals contain two additional Y-family polymerases. Polt is the only known DNA polymerase to date that violates the Watson-Crick base-pairing rule [106]. It relies on Hoogsteen base pairing as opposed to typical WatsonCrick base pairing and thus operates with very low fidelity [107]. This mechanism may facilitate read-through of replication-blocking minor groove purine adducts [108]. In vivo, uracil derived from cytosine deamination may be the desired target of Poll as it inserts a G opposite a template U [109].

Polк is thought to be involved in the elongation step following mismatched bases or following damaged bases [110-112], and reads through bulky adducts such as modifications by benzo[a]pyrene diol epoxide (BPDE) [113, $114]$. When nucleotides containing dG- $N^{2}$-BPDE, the most potent carcinogenic compound produced by industrial and cigarette smoke, are used as template, Polк can bypass the adduct with much higher efficiency than Pol $\eta$ or Polı by correctly inserting C opposite the bulky lesion [114]. However, when undamaged DNA or DNA containing some common

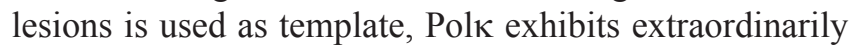
low fidelity [115-117].

In summary, although each of its members has distinct base-pair specificity and specialized functions, Y-family polymerases are highly conserved (Figure 3 ) and generally allow significantly reduced base-pair fidelity and thus result in elevated mutagenic potential. The low fidelity of these polymerases suggests that their activities must be restricted to highly selective conditions in order to limit mutational events.

\section{Regulated access of Y-family polymerases to the damage site}

Because of the high probability of TLS polymerases being mutagenic, it is expected that these polymerases are tightly regulated, probably at different levels. At the transcriptional level, an example is that human and mouse $P O L K$ promoters contain xenobiotic responsive elements (XREs) that can be induced by polycyclic aromatic hydrocarbons (PAHs), among which benzo $[a]$ pyrene is the most characterized [118]. Hence, POLK is induced in response to specific DNA damage that can be bypassed by Polк. The second regulatory mechanism is damage-induced accumulation at the replication foci stalled at DNA damage. Poln forms such foci in response to UV irradiation, and mutations with a functional polymerase motif but lacking the domain for relocalization into the damage foci are found in the XPV patient [119]. Polt physically interacts and colocalizes with Poln to the damage-induced nuclear foci [120], suggesting that Polt plays a role in bypassing UV-induced lesions. In contrast, BPDE treatment specifi-

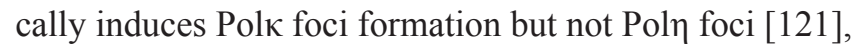
indicating lesion-specific recruitment of the cognate Yfamily polymerase. The mechanism of this lesion-specific recruitment is currently unknown.

Perhaps the most exciting advance in recent years is the discovery that all eukaryotic Y-family polymerases contain both PCNA interacting peptide (PIP) and Ub-binding domains, including Ub-binding motifs (UBMs) or Ub-binding zinc fingers (UBZs) (Figure 3). Poln specifically interacts with monoubiquitinated but not unmodified PCNA [122], and the Ub-binding domains are essential for the accumulation of Polı and Pol $\eta$ in replication foci. Similarly, the damage-induced foci formation and UV resistance of Rev1 also requires UBMs [123]. Unlike other Y-family polymerases, Rev1 does not contain a PIP motif; instead, a recent study 
suggests that Rev1 utilizes its BRCT domain to interact with PCNA [95]. Supporting this notion is the observation that the damage-induced foci formation of Pol $\eta[122,124]$ and Polк [125] is dependent on functional Rad18, presumably because Rad 18 is required for the generation of monoubiquitinated PCNA. An ultimate support perhaps comes from an in vitro study [126], in which PCNA was found to be only ubiquitinated when appropriately loaded onto DNA. The ubiquitinated PCNA shows similar functional interactions as unmodified PCNA with replication factors such as Fen1, Lig1, RFC, Pol $\delta$ and Pole, but, in addition, is able to activate Pol $\eta$ and Rev1 [126].

Although the above studies provide a paradigm for the restriction and recruitment of TLS polymerases to the damage site, the overall model is challenged by other reports. One study shows that unmodified PCNA is sufficient to stimulate DNA synthesis by Polк, primarily by reducing the $K m$ to enhance correct nucleotide incorporation [127]. The direct challenge came from the in vitro reconstitution of the DNA synthesis reaction, in which PCNA monoubiquitinated on all three monomers does not enhance affinity for any polymerases examined, nor does it enhance TLS activity by Y-family polymerases [128]. Furthermore, a recent report [129] showed that mutations in the UBZ motif of yeast Pol $\eta$ did not impair its in vivo or in vitro TLS functions. The authors suggested an alternative model in which PCNA monoubiquitination may disrupt its interactions with a protein(s) that inhibits binding to the TLS polymerases. To date no such candidate protein has been identified, although we notice a recent report [130] that Mgs1, a protein with homology to E. coli RuvB and eukaryotic clamp loader protein RFC, as well as DNA-dependent ATPase activity and DNA-annealing activities $[131,132]$, associates with PCNA and appears to repress the RAD6 pathway in the absence of exogenous damage. Other concerns with the above paradigm include the stability of monoubiquitinated PCNA, particularly in mammalian cells, that extends past the expected time required to bypass the damage [122], which would allow persistent TLS with unnecessarily increased mutation rates. In addition, hydroxyurea treatment, which depletes the nucleotide pool and induces replication fork stalling, also results in PCNA monoubiquitination [120]. The stalled replication fork after this treatment is unlikely to benefit from TLS, raising doubt that mono-Ub is at the heart of polymerase switching.

PCNA modification may not be the only means of promoting TLS; DNA damage checkpoints have been implicated in TLS. For instance, Rad9 of the $S$. pombe 9-1-1 complex, which forms a PCNA-like heterotrimeric clamp, associates with Mms2, and a mutant form of Rad9 incapable of interaction promotes mutagenesis in a TLS-dependent manner [133]. In budding yeast the phosphorylation by protein kinase Mec1 induces the re-localization of Rev1 and Pol $\xi$ to sites of DNA double-strand breaks independently of mono-Ub PCNA [134]. Furthermore, the budding yeast 9-1-1 clamp physically interacts with the Rev7 subunit of Pol $\zeta$ and is partially required for spontaneous mutagenesis in a Pol $\zeta$-dependent manner [135]. We wish to emphasize that the above observations did not directly conflict with the PCNA-TLS model.

\section{Error-free DDT}

Despite the advances made with PCNA and TLS in the past years, little is known about the molecular events leading to error-free DDT following PCNA polyubiquitination. Apparently, the error-free bypass has to utilize newly synthesized sister chromatid as a template, and, much like PCNA mono-Ub, poly-Ub of PCNA may provide a signal to initiate the process. Two possible models, namely template switching and replication fork regression, have been proposed [5]. Template switching involves homologous sister chromatid invasion/cohesion, high-fidelity DNA synthesis and the subsequent resolution of a Holliday junction (Figure 1). Fork regression (Figure 1) is thought to operate much as it does in bacteria, requiring ssDNA binding protein and RecA to produce a characteristic chicken-foot structure [15]. Experimental evidence to support a chicken-foot structure in eukaryotes came from a recent report [136] that yeast Rad5 has a DNA helicase activity that facilitates replication fork regression. In contrast, several recent reviews [137-139] suggest that the DNA damage checkpoint acts to prevent stalled replication fork regression, while error-free DDT is mediated by template switching. Alternatively, the two error-free DDT models may not be mechanistically different as they appear. Regardless of the mode of reaction, it is abundantly clear that the error-free DDT process is highly conserved in the entire eukaryotic kingdom, from yeast to human. Sequence and functional homologs of all proteins involved in error-free DDT, including Mms2 [140], Ubc13 [141] and Rad5 [142, 143], have been found in mammals, plants and other higher eukaryotes [144, 145]. For a few limited examples, suppression of the above genes resulted in phenotypes reminiscent of the corresponding yeast mutants [143, 146, 147].

\section{DDT, genomic instability and cancer}

Studies in the yeast model have clearly demonstrated the significance of DDT in maintaining genomic stability. The two branches within DDT, with one being highly mutagenic and another error-free, are likely kept to a dynamic balance in wild-type cells. However, in yeast cells defective in error-free DDT, spontaneous mutation rates can be elevated 
by 30 -fold, which would be viewed as a predisposition to cancer. It was postulated that error-prone TLS may constitute a major source of genomic instability and cancer [148], although direct evidence is rather lacking.

Perhaps the best studied example of TLS and tumorigenesis is the discovery of mammalian Pol $\eta$, whose gene was found mutated in all XPV patients examined. Pol $\eta$ co-localizes with Rev1 [149], Polt [120] and mono-Ub PCNA [124], suggesting that mutations in these genes may also be associated with cancer. The level of translesion polymerases in normal and matched tumor cell lines has been investigated. Several lung cancer cell lines were found to overexpress

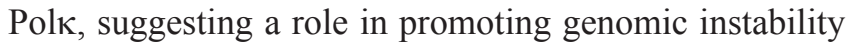
and cancer [150]. In another study, however, transcript levels of TLS polymerases $\eta, \imath, \kappa$ and $\zeta$ are significantly reduced in various lung, stomach and colorectal cancers [151]. Clearly, more research is required to establish roles of DDT in tumorigenesis and carcinogenesis.

\section{Acknowledgments}

The authors wish to thank Landon Pastushok, Michelle Hanna and other members from the Xiao laboratory for helpful discussion. This work was supported by the Canadian Institutes of Health Research operating grants MOP-38104 and MOP-53240 to W Xiao, and the National Natural Science Foundation of China(Grant no. 30560132) to $\mathrm{F} \mathrm{Xu}$.

\section{References}

1 Rupp WD, Howard-Flanders P. Discontinuities in the DNA synthesized in an excision-defective strain of Escherichia coli following ultraviolet irradiation. J Mol Biol 1968; 31:291304.

2 Cordeiro-Stone M, Makhov AM, Zaritskaya LS, Griffith JD. Analysis of DNA replication forks encountering a pyrimidine dimer in the template to the leading strand. J Mol Biol 1999; 289:1207-1218.

3 Bridges BA, Munson RJ. Mutagenesis in Escherichia coli: evidence for the mechanism of base change mutation by ultraviolet radiation in a strain deficient in excision-repair. Proc $R$ Soc Lond B Biol Sci 1968; 171:213-226.

4 Ganesan AK. Persistence of pyrimidine dimers during postreplication repair in ultraviolet light-irradiated Escherichia coli K12. J Mol Biol 1974; 87:103-119.

5 Broomfield S, Hryciw T, Xiao W. DNA postreplication repair and mutagenesis in Saccharomyces cerevisiae. Mutat Res 2001; 486:167-184.

6 Barbour L, Xiao W. Regulation of alternative replication bypass pathways at stalled replication forks and its effects on genome stability: a yeast model. Mutat Res 2003; 532:137-155.

7 Moldovan GL, Pfander B, Jentsch S. PCNA, the maestro of the replication fork. Cell 2007; 129:665-679.

8 Bridges BA, Sedgwick SG. Effect of photoreactivation on the filling of gaps in deoxyribonucleic acid synthesized after expo- sure of Escherichia coli to ultraviolet light. J Bacteriol 1974; 117:1077-1081.

9 Howard-Flanders P, Rupp WD, Wilkins BM, Cole RS. DNA replication and recombination after UV irradiation. Cold Spring Harb Symp Quant Biol 1968; 33:195-207.

10 Salles B, Defais M. Signal of induction of RecA protein in $E$. coli. Mutat Res 1984; 131:53-59.

11 Little JW. Autodigestion of LexA and phage lambda repressors. Proc Natl Acad Sci USA 1984; 81:1375-1379.

12 Reuven NB, Arad G, Maor-Shoshani A, Livneh Z. The mutagenesis protein UmuC is a DNA polymerase activated by UmuD', RecA, and SSB and is specialized for translesion replication. $J$ Biol Chem 1999; 274:31763-31766.

13 Tang M, Bruck I, Eritja R, et al. Biochemical basis of SOSinduced mutagenesis in Escherichia coli: reconstitution of in vitro lesion bypass dependent on the UmuD'2C mutagenic complex and RecA protein. Proc Natl Acad Sci USA 1998; 95:9755-9760.

14 Pham P, Seitz EM, Saveliev S, et al. Two distinct modes of RecA action are required for DNA polymerase $\mathrm{V}$-catalyzed translesion synthesis. Proc Natl Acad Sci USA 2002; 99:11061-11066.

15 Robu ME, Inman RB, Cox MM. RecA protein promotes the regression of stalled replication forks in vitro. Proc Natl Acad Sci USA 2001; 98:8211-8218.

16 Hochstrasser M. Ubiquitin-dependent protein degradation. Annu Rev Genet 1996; 30:405-439.

17 Pickart CM. Back to the future with ubiquitin. Cell 2004; 116:181-190.

18 Pickart CM, Fushman D. Polyubiquitin chains: polymeric protein signals. Curr Opin Chem Biol 2004; 8:610-616.

19 Hochstrasser M. Evolution and function of ubiquitin-like protein-conjugation systems. Nat Cell Biol 2000; 2:E153-E157.

20 Schwartz DC, Hochstrasser M. A superfamily of protein tags: ubiquitin, SUMO and related modifiers. Trends Biochem Sci 2003; 28:321-328.

21 Bayer P, Arndt A, Metzger S, et al. Structure determination of the small ubiquitin-related modifier SUMO-1. J Mol Biol 1998; 280:275-286.

22 Sheng W, Liao X. Solution structure of a yeast ubiquitin-like protein Smt3: the role of structurally less defined sequences in protein-protein recognitions. Protein Sci 2002; 11:1482-1491.

23 Prakash L. Characterization of postreplication repair in Saccharomyces cerevisiae and effects of rad6, rad18, rev3 and rad52 mutations. Mol Gen Genet 1981; 184:471-478.

24 Haynes RH, Kunz BA. DNA repair and mutagenesis in yeast. In: Strathern J, Jones EW, Broach JR (Eds.) The Molecular Biology of the Yeast Saccharomyces cerevisiae: Life Cycle and Inheritance. Cold Spring Harbor, NY: Cold Spring Harbor Laboratory Press, 1981, 371-414.

25 Friedberg EC. Deoxyribonucleic acid repair in the yeast Saccharomyces cerevisiae. Microbiol Rev 1988; 52:70-102.

26 Prakash S, Sung P, Prakash L. DNA repair genes and proteins of Saccharomyces cerevisiae. Annu Rev Genet 1993; 27:33-70.

27 Montelone BA, Prakash S, Prakash L. Recombination and mutagenesis in rad6 mutants of Saccharomyces cerevisiae: evidence for multiple functions of the RAD6 gene. Mol Gen Genet 1981; 184:410-415.

28 Jentsch S, McGrath JP, Varshavsky A. The yeast DNA repair gene RAD6 encodes a ubiquitin-conjugating enzyme. Nature 1987; 329:131-134. 
29 Sung P, Berleth E, Pickart C, Prakash S, Prakash L. Yeast RAD6 encoded ubiquitin conjugating enzyme mediates protein degradation dependent on the N-end-recognizing E3 enzyme. EMBO $J$ 1991; 10:2187-2193.

30 Sung P, Prakash S, Prakash L. The RAD6 protein of Saccharomyces cerevisiae polyubiquitinates histones, and its acidic domain mediates this activity. Genes Dev 1988; 2:1476-1485.

31 Robzyk K, Recht J, Osley MA. Rad6-dependent ubiquitination of histone H2B in yeast. Science 2000; 287:501-504.

32 Morrison A, Miller EJ, Prakash L. Domain structure and functional analysis of the carboxyl-terminal polyacidic sequence of the RAD6 protein of Saccharomyces cerevisiae. Mol Cell Biol 1988; 8:1179-1185.

33 Reynolds P, Weber S, Prakash L. RAD6 gene of Saccharomyces cerevisiae encodes a protein containing a tract of 13 consecutive aspartates. Proc Natl Acad Sci USA 1985; 82:168-172.

34 Reynolds P, Koken MH, Hoeijmakers JH, Prakash S, Prakash L. The rhp6+ gene of Schizosaccharomyces pombe: a structural and functional homolog of the RAD6 gene from the distantly related yeast Saccharomyces cerevisiae. EMBO J 1990; 9:1423-1430.

35 Koken M, Reynolds P, Bootsma D, Hoeijmakers J, Prakash S, Prakash L. Dhr6, a Drosophila homolog of the yeast DNA-repair gene RAD6. Proc Natl Acad Sci USA 1991; 88:3832-3836.

36 Koken MH, Reynolds P, Jaspers-Dekker I, Prakash L, Prakash S, Bootsma D, Hoeijmakers JH. Structural and functional conservation of two human homologs of the yeast DNA repair gene RAD6. Proc Natl Acad Sci USA 1991; 88:8865-8869.

37 Watkins JF, Sung P, Prakash S, Prakash L. The extremely conserved amino terminus of RAD6 ubiquitin-conjugating enzyme is essential for amino-end rule-dependent protein degradation. Genes Dev 1993; 7:250-261.

38 Dohmen RJ, Madura K, Bartel B, Varshavsky A. The N-end rule is mediated by the UBC2(RAD6) ubiquitin-conjugating enzyme. Proc Natl Acad Sci USA 1991; 88:7351-7355.

39 Bailly V, Lamb J, Sung P, Prakash S, Prakash L. Specific complex formation between yeast RAD6 and RAD18 proteins: a potential mechanism for targeting RAD6 ubiquitin-conjugating activity to DNA damage sites. Genes Dev 1994; 8:811-820.

40 Bailly V, Lauder S, Prakash S, Prakash L. Yeast DNA repair proteins Rad6 and Rad18 form a heterodimer that has ubiquitin conjugating, DNA binding, and ATP hydrolytic activities. J Biol Chem 1997; 272:23360-23365.

41 Lovering R, Hanson IM, Borden KL, et al. Identification and preliminary characterization of a protein motif related to the zinc finger. Proc Natl Acad Sci USA 1993; 90:2112-2116.

42 Saurin AJ, Borden KL, Boddy MN, Freemont PS. Does this have a familiar RING? Trends Biochem Sci 1996; 21:208-214.

43 Hoege C, Pfander B, Moldovan GL, Pyrowolakis G, Jentsch S. RAD6-dependent DNA repair is linked to modification of PCNA by ubiquitin and SUMO. Nature 2002; 419:135-141.

44 Jones JS, Weber S, Prakash L. The Saccharomyces cerevisiae $R A D 18$ gene encodes a protein that contains potential zinc finger domains for nucleic acid binding and a putative nucleotide binding sequence. Nucleic Acids Res 1988; 16:7119-7131.

45 Kunz BA, Kang XL, Kohalmi L. The yeast rad18 mutator specifically increases $\mathrm{G}-\mathrm{C}-\mathrm{T}-\mathrm{A}$ transversions without reducing correction of G-A or C-T mismatches to G-C pairs. Mol Cell Biol 1991; 11:218-225.

46 Lemontt JF. Pathways of ultraviolet mutability in Saccharomyces cerevisiae. II. The effect of rev genes on recombination. Mutat Res 1971; 13:319-326.

47 Lemontt JF. Mutants of yeast defective in mutation by ultraviolet light. Genetics 1971; 68:21-33.

48 Larimer FW, Perry JR, Hardigree AA. The REV1 gene of Saccharomyces cerevisiae: isolation, sequence, and functional analysis. J Bacteriol 1989; 171:230-237.

49 Nelson JR, Lawrence CW, Hinkle DC. Deoxycytidyl transferase activity of yeast REV1 protein. Nature 1996; 382:729-731.

50 Nelson JR, Lawrence CW, Hinkle DC. Thymine-thymine dimer bypass by yeast DNA polymerase $\zeta$. Science 1996; 272:16461649.

51 Lawrence CW. Cellular functions of DNA polymerase $\zeta$ and Rev1 protein. Adv Protein Chem 2004; 69:167-203.

52 Broomfield S, Chow BL, Xiao W. MMS2, encoding a ubiquitin-conjugating-enzyme-like protein, is a member of the yeast error-free postreplication repair pathway. Proc Natl Acad Sci USA 1998; 95:5678-5683.

53 Xiao W, Chow BL, Fontanie T, et al. Genetic interactions between error-prone and error-free postreplication repair pathways in Saccharomyces cerevisiae. Mutat Res 1999; 435:1-11.

54 Hofmann RM, Pickart CM. Noncanonical MMS2-encoded ubiquitin-conjugating enzyme functions in assembly of novel polyubiquitin chains for DNA repair. Cell 1999; 96:645-653.

55 Brusky J, Zhu Y, Xiao W. UBC13, a DNA-damage-inducible gene, is a member of the error-free postreplication repair pathway in Saccharomyces cerevisiae. Curr Genet 2000; 37:168174.

56 Ulrich HD, Jentsch S. Two RING finger proteins mediate cooperation between ubiquitin-conjugating enzymes in DNA repair. EMBO J 2000; 19:3388-3397.

57 Johnson RE, Henderson ST, Petes TD, Prakash S, Bankmann M, Prakash L. Saccharomyces cerevisiae RAD5-encoded DNA repair protein contains DNA helicase and zinc-binding sequence motifs and affects the stability of simple repetitive sequences in the genome. Mol Cell Biol 1992; 12:3807-3818.

58 Johnson RE, Prakash S, Prakash L. Yeast DNA repair protein RAD5 that promotes instability of simple repetitive sequences is a DNA-dependent ATPase. J Biol Chem 1994; 269:2825928262.

59 Ahne F, Jha B, Eckardt-Schupp F. The RAD5 gene product is involved in the avoidance of non-homologous end-joining of DNA double strand breaks in the yeast Saccharomyces cerevisiae. Nucleic Acids Res 1997; 25:743-749.

60 Chen S, Davies AA, Sagan D, Ulrich HD. The RING finger ATPase Rad5p of Saccharomyces cerevisiae contributes to DNA double-strand break repair in a ubiquitin-independent manner. Nucleic Acids Res 2005; 33:5878-5886.

61 Torres-Ramos CA, Yoder BL, Burgers PM, Prakash S, Prakash L. Requirement of proliferating cell nuclear antigen in RAD6dependent postreplicational DNA repair. Proc Natl Acad Sci USA 1996; 93:9676-9681.

62 Garg P, Burgers PM. Ubiquitinated proliferating cell nuclear antigen activates translesion DNA polymerases $\eta$ and REV1. Proc Natl Acad Sci USA 2005; 102:18361-18366.

63 Stelter P, Ulrich HD. Control of spontaneous and damage-induced mutagenesis by SUMO and ubiquitin conjugation. Nature 2003; 425:188-191.

64 Moldovan GL, Pfander B, Jentsch S. PCNA controls establish- 
ment of sister chromatid cohesion during S phase. Mol Cell 2006; 23:723-732.

65 Lawrence CW, Christensen RB. Metabolic suppressors of trimethoprim and ultraviolet light sensitivities of Saccharomyces cerevisiae rad6 mutants. J Bacteriol 1979; 139:866-876.

66 Rong L, Klein HL. Purification and characterization of the SRS2 DNA helicase of the yeast Saccharomyces cerevisiae. J Biol Chem 1993; 268:1252-1259.

67 Rong L, Palladino F, Aguilera A, Klein HL. The hyper-gene conversion hpr5-1 mutation of Saccharomyces cerevisiae is an allele of the SRS2/RADH gene. Genetics 1991; 127:75-85.

68 Broomfield S, Xiao W. Suppression of genetic defects within the $R A D 6$ pathway by $s r s 2$ is specific for error-free post-replication repair but not for damage-induced mutagenesis. Nucleic Acids Res 2002; 30:732-739.

69 Ulrich HD. The $s r s 2$ suppressor of UV sensitivity acts specifically on the RAD5- and MMS2-dependent branch of the RAD6 pathway. Nucleic Acids Res 2001; 29:3487-3494.

70 Aboussekhra A, Chanet R, Zgaga Z, Cassier-Chauvat C, Heude M, Fabre F. RADH, a gene of Saccharomyces cerevisiae encoding a putative DNA helicase involved in DNA repair. Characteristics of radH mutants and sequence of the gene. Nucleic Acids Res 1989; 17:7211-7219.

71 Aguilera A, Klein HL. Genetic control of intrachromosomal recombination in Saccharomyces cerevisiae. I. Isolation and genetic characterization of hyper-recombination mutations. Genetics 1988; 119:779-790.

72 Chanet R, Heude M, Adjiri A, Maloisel L, Fabre F. Semidominant mutations in the yeast Rad51 protein and their relationships with the Srs2 helicase. Mol Cell Biol 1996; 16:4782-4789.

73 Kaytor MD, Nguyen M, Livingston DM. The complexity of the interaction between RAD52 and SRS2. Genetics 1995; 140:14411442.

74 Milne GT, Ho T, Weaver DT. Modulation of Saccharomyces cerevisiae DNA double-strand break repair by SRS2 and RAD51. Genetics 1995; 139:1189-1199.

75 Schild D. Suppression of a new allele of the yeast RAD52 gene by overexpression of RAD51, mutations in $s r s 2$ and $c c r 4$, or mating-type heterozygosity. Genetics 1995; 140:115-127.

76 Veaute X, Jeusset J, Soustelle C, Kowalczykowski SC, Le Cam E, Fabre F. The Srs2 helicase prevents recombination by disrupting Rad51 nucleoprotein filaments. Nature 2003; 423:309-312.

77 Krejci L, Van Komen S, Li Y, et al. DNA helicase Srs2 disrupts the Rad51 presynaptic filament. Nature 2003; 423:305-309.

78 Pfander B, Moldovan GL, Sacher M, Hoege C, Jentsch S. SUMO-modified PCNA recruits Srs2 to prevent recombination during S phase. Nature 2005; 436:428-433.

79 Papouli E, Chen S, Davies AA, et al. Crosstalk between SUMO and ubiquitin on PCNA is mediated by recruitment of the helicase Srs2p. Mol Cell 2005; 19:123-133.

80 Haracska L, Torres-Ramos CA, Johnson RE, Prakash S, Prakash L. Opposing effects of ubiquitin conjugation and SUMO modification of PCNA on replicational bypass of DNA lesions in Saccharomyces cerevisiae. Mol Cell Biol 2004; 24:4267-4274.

81 Friedberg EC, Walker GC, Siede W, Wood RD, Schultz RA, Ellenberger T. DNA Repair and Mutagenesis, 2nd ed. Washington, DC: ASM Press, 2006.

82 Yang W, Woodgate R. What a difference a decade makes: in- sights into translesion DNA synthesis. Proc Natl Acad Sci USA 2007; 104:15591-15598.

83 Woodgate R. A plethora of lesion-replicating DNA polymerases. Genes Dev 1999; 13:2191-2195.

84 Wang Z. Translesion synthesis by the UmuC family of DNA polymerases. Mutat Res 2001; 486:59-70.

85 Lehmann AR, Niimi A, Ogi T, et al. Translesion synthesis: Yfamily polymerases and the polymerase switch. DNA Repair (Amst) 2007; 6:891-899.

86 Friedberg EC, Gerlach VL. Novel DNA polymerases offer clues to the molecular basis of mutagenesis. Cell 1999; 98:413-416.

87 Wagner J, Fujii S, Gruz P, Nohmi T, Fuchs RP. The beta clamp targets DNA polymerase IV to DNA and strongly increases its processivity. EMBO Rep 2000; 1:484-488.

88 Tang M, Pham P, Shen X, Taylor JS, O'Donnell M, Woodgate $\mathrm{R}$, et al. Roles of $E$. coli DNA polymerases IV and V in lesion-targeted and untargeted SOS mutagenesis. Nature 2000; 404:1014-1018.

89 Kobayashi S, Valentine MR, Pham P, O’Donnell M, Goodman MF. Fidelity of Escherichia coli DNA polymerase IV. Preferential generation of small deletion mutations by dNTP-stabilized misalignment. J Biol Chem 2002; 277:34198-34207.

90 Fiala KA, Suo Z. Mechanism of DNA polymerization catalyzed by Sulfolobus solfataricus P2 DNA polymerase IV. Biochemistry 2004; 43:2116-2125.

91 Fuchs RP, Fujii S, Wagner J. Properties and functions of Escherichia coli: Pol IV and Pol V. Adv Protein Chem 2004; 69:229264.

92 Nelson JR, Gibbs PE, Nowicka AM, Hinkle DC, Lawrence CW. Evidence for a second function for Saccharomyces cerevisiae Rev1p. Mol Microbiol 2000; 37:549-554.

93 Nair DT, Johnson RE, Prakash L, Prakash S, Aggarwal AK. Rev1 employs a novel mechanism of DNA synthesis using a protein template. Science 2005; 309:2219-2222.

94 Otsuka C, Kunitomi N, Iwai S, Loakes D, Negishi K. Roles of the polymerase and BRCT domains of Rev1 protein in translesion DNA synthesis in yeast in vivo. Mutat Res 2005; 578:79-87.

95 Guo C, Sonoda E, Tang TS, et al. REV1 protein interacts with PCNA: significance of the REV1 BRCT domain in vitro and in vivo. Mol Cell 2006; 23:265-271.

96 Acharya N, Haracska L, Johnson RE, Unk I, Prakash S, Prakash L. Complex formation of yeast Rev1 and Rev7 proteins: a novel role for the polymerase-associated domain. Mol Cell Biol 2005; 25:9734-9740.

97 Guo C, Fischhaber PL, Luk-Paszyc MJ, et al. Mouse Rev1 protein interacts with multiple DNA polymerases involved in translesion DNA synthesis. EMBO J 2003; 22:6621-6630.

98 Gibbs PE, Wang XD, Li Z, McManus TP, McGregor WG, Lawrence $\mathrm{CW}$, et al. The function of the human homolog of Saccharomyces cerevisiae REV1 is required for mutagenesis induced by UV light. Proc Natl Acad Sci USA 2000; 97:41864191.

99 McDonald JP, Levine AS, Woodgate R. The Saccharomyces cerevisiae RAD30 gene, a homologue of Escherichia coli dinB and $u m u C$, is DNA damage inducible and functions in a novel error-free postreplication repair mechanism. Genetics 1997; 147:1557-1568.

100 Masutani C, Kusumoto R, Yamada A, et al. The XPV (xeroderma pigmentosum variant) gene encodes human DNA polymerase 
ๆ. Nature 1999; 399:700-704.

101 Johnson RE, Kondratick CM, Prakash S, Prakash L. hRAD30 mutations in the variant form of xeroderma pigmentosum. Science 1999; 285:263-265.

102 Johnson RE, Prakash S, Prakash L. Efficient bypass of a thymine-thymine dimer by yeast DNA polymerase, Polh. Science 1999; 283:1001-1004.

103 Johnson RE, Washington MT, Prakash S, Prakash L. Fidelity of human DNA polymerase $\eta$. J Biol Chem 2000; 275:74477450 .

104 Washington MT, Prakash L, Prakash S. Yeast DNA polymerase $\eta$ utilizes an induced-fit mechanism of nucleotide incorporation. Cell 2001; 107:917-927.

105 Vaisman A, Lehmann AR, Woodgate R. DNA polymerases $\mathrm{h}$ and i. Adv Protein Chem 2004; 69:205-228.

106 Tissier A, McDonald JP, Frank EG, Woodgate R. poli, a remarkably error-prone human DNA polymerase. Genes Dev 2000; 14:1642-1650.

107 Nair DT, Johnson RE, Prakash S, Prakash L, Aggarwal AK. Replication by human DNA polymerase-i occurs by Hoogsteen base-pairing. Nature 2004; 430:377-380.

108 Wolfle WT, Johnson RE, Minko IG, Lloyd RS, Prakash S, Prakash L. Human DNA polymerase 1 promotes replication through a ring-closed minor-groove adduct that adopts a syn conformation in DNA. Mol Cell Biol 2005; 25:8748-8754.

109 Vaisman A, Woodgate R. Unique misinsertion specificity of polt may decrease the mutagenic potential of deaminated cytosines. EMBO J 2001; 20:6520-6529.

110 Haracska L, Prakash L, Prakash S. Role of human DNA polymerase $\kappa$ as an extender in translesion synthesis. Proc Natl Acad Sci USA 2002; 99:16000-16005.

111 Wolfle WT, Washington MT, Prakash L, Prakash S. Human DNA polymerase $\kappa$ uses template-primer misalignment as a novel means for extending mispaired termini and for generating single-base deletions. Genes Dev 2003; 17:2191-2199.

112 Ohmori H, Ohashi E, Ogi T. Mammalian Pol $\kappa$ : regulation of its expression and lesion substrates. Adv Protein Chem 2004; 69:265-278.

113 Shen X, Sayer JM, Kroth H, et al. Efficiency and accuracy of SOS-induced DNA polymerases replicating benzo[ $a]$ pyrene7,8-diol 9,10-epoxide A and G adducts. J Biol Chem 2002; 277:5265-5274.

114 Ogi T, Shinkai Y, Tanaka K, Ohmori H. Polk protects mammalian cells against the lethal and mutagenic effects of benzo $[a]$ pyrene. Proc Natl Acad Sci USA 2002; 99:15548-15553.

115 Ohashi E, Ogi T, Kusumoto R, Iwai S, Masutani C, Hanaoka F, et al. Error-prone bypass of certain DNA lesions by the human DNA polymerase . Genes Dev 2000; 14:1589-1594.

116 Zhang Y, Yuan F, Wu X, et al. Error-free and error-prone lesion bypass by human DNA polymerase $\kappa$ in vitro. Nucleic Acids Res 2000; 28:4138-4146.

117 Zhang Y, Yuan F, Xin H, et al. Human DNA polymerase $\kappa$ synthesizes DNA with extraordinarily low fidelity. Nucleic Acids Res 2000; 28:4147-4156.

118 Ogi T, Mimura J, Hikida M, Fujimoto H, Fujii-Kuriyama Y, Ohmori H. Expression of human and mouse genes encoding polk: testis-specific developmental regulation and AhR-dependent inducible transcription. Genes Cells 2001; 6:943-953.

119 Kannouche P, Broughton BC, Volker M, Hanaoka F, Mullenders
LH, Lehmann AR. Domain structure, localization, and function of DNA polymerase $\eta$, defective in xeroderma pigmentosum variant cells. Genes Dev 2001; 15:158-172.

120 Kannouche P, Fernandez de Henestrosa AR, Coull B, et al. Localization of DNA polymerases $\eta$ and $\imath$ to the replication machinery is tightly co-ordinated in human cells. EMBO J 2002; 21:6246-6256.

$121 \mathrm{Bi}$ X, Slater DM, Ohmori H, Vaziri C. DNA polymerase $\kappa$ is specifically required for recovery from the benzo $[a]$ pyrene-dihydrodiol epoxide (BPDE)-induced S-phase checkpoint. J Biol Chem 2005; 280:22343-22355.

122 Kannouche PL, Wing J, Lehmann AR. Interaction of human DNA polymerase $\eta$ with monoubiquitinated PCNA: a possible mechanism for the polymerase switch in response to DNA damage. Mol Cell 2004; 14:491-500.

123 Guo C, Tang TS, Bienko M, et al. Ubiquitin-binding motifs in REV1 protein are required for its role in the tolerance of DNA damage. Mol Cell Biol 2006; 26:8892-8900.

124 Watanabe K, Tateishi S, Kawasuji M, Tsurimoto T, Inoue H, Yamaizumi M. Rad18 guides polh to replication stalling sites through physical interaction and PCNA monoubiquitination. EMBO J 2004; 23:3886-3896.

125 Bi X, Barkley LR, Slater DM, et al. Rad18 regulates DNA polymerase $\kappa$ and is required for recovery from S-phase checkpoint-mediated arrest. Mol Cell Biol 2006; 26:3527-3540.

126 Garg P, Burgers PM. Ubiquitinated proliferating cell nuclear antigen activates translesion DNA polymerases $\eta$ and REV1. Proc Natl Acad Sci USA 2005; 102:18361-18366.

127 Haracska L, Unk I, Johnson RE, et al. Stimulation of DNA synthesis activity of human DNA polymerase $\kappa$ by PCNA. Mol Cell Biol 2002; 22:784-791.

128 Haracska L, Unk I, Prakash L, Prakash S. Ubiquitylation of yeast proliferating cell nuclear antigen and its implications for translesion DNA synthesis. Proc Natl Acad Sci USA 2006; 103:6477-6482.

129 Acharya N, Brahma A, Haracska L, Prakash L, Prakash S. Mutations in the ubiquitin binding UBZ motif of DNA polymerase $\eta$ do not impair its function in translesion synthesis during replication. Mol Cell Biol 2007; 27:7266-7272.

130 Hishida T, Ohya T, Kubota Y, Kamada Y, Shinagawa H. Functional and physical interaction of yeast Mgs1 with PCNA: impact on RAD6-dependent DNA damage tolerance. Mol Cell Biol 2006; 26:5509-5517.

131 Hishida T, Iwasaki H, Ohno T, Morishita T, Shinagawa H. A yeast gene, $M G S 1$, encoding a DNA-dependent AAA(+) ATPase is required to maintain genome stability. Proc Natl Acad Sci USA 2001; 98:8283-8289.

132 Hishida T, Ohno T, Iwasaki H, Shinagawa H. Saccharomyces cerevisiae MGS1 is essential in strains deficient in the RAD6dependent DNA damage tolerance pathway. EMBO J 2002; 21:2019-2029.

133 Kai M, Furuya K, Paderi F, Carr AM, Wang TS. Rad3-dependent phosphorylation of the checkpoint clamp regulates repair-pathway choice. Nat Cell Biol 2007; 9:691-697.

134 Hirano Y, Sugimoto K. ATR homolog Mec1 controls association of DNA polymerase $\zeta$-Rev1 complex with regions near a double-strand break. Curr Biol 2006; 16:586-590.

135 Sabbioneda S, Minesinger BK, Giannattasio M, Plevani P, Muzi-Falconi M, Jinks-Robertson S. The 9-1-1 checkpoint 
clamp physically interacts with polz and is partially required for spontaneous pol $\zeta$-dependent mutagenesis in Saccharomyces cerevisiae. J Biol Chem 2005; 280:38657-38665.

136 Blastyak A, Pinter L, Unk I, Prakash L, Prakash S, Haracska L. Yeast Rad5 protein required for postreplication repair has a DNA helicase activity specific for replication fork regression. Mol Cell 2007; 28:167-175.

137 Cobb JA, Bjergbaek L. RecQ helicases: lessons from model organisms. Nucleic Acids Res 2006; 34:4106-4114.

138 Branzei D, Foiani M. Interplay of replication checkpoints and repair proteins at stalled replication forks. DNA Repair (Amst) 2007; 6:994-1003.

139 Klein HL. A SUMOry of DNA replication: synthesis, damage, and repair. Cell 2006; 127:455-457.

140 Xiao W, Lin SL, Broomfield S, Chow BL, Wei YF. The products of the yeast MMS2 and two human homologs ( $h M M S 2$ and $C R O C-1)$ define a structurally and functionally conserved Ubclike protein family. Nucleic Acids Res 1998; 26:3908-3914.

141 Oh CE, McMahon R, Benzer S, Tanouye MA. bendless, a Drosophila gene affecting neuronal connectivity, encodes a ubiquitin-conjugating enzyme homolog. J Neurosci 1994; 14:3166-3179.

142 Unk I, Hajdu I, Fatyol K, et al. Human SHPRH is a ubiquitin ligase for Mms2-Ubc13-dependent polyubiquitylation of proliferating cell nuclear antigen. Proc Natl Acad Sci USA 2006; 103:18107-18112.

143 Motegi A, Sood R, Moinova H, Markowitz SD, Liu PP, Myung K. Human SHPRH suppresses genomic instability through proliferating cell nuclear antigen polyubiquitination. J Cell Biol 2006; 175:703-708.

144 Pastushok L, Xiao W. DNA postreplication repair modulated by ubiquitination and sumoylation. Adv Protein Chem 2004; 69:279-306.

145 Kunz BA, Xiao W. DNA damage tolerance in plants via translesion synthesis. Genes, Genomes Genomics 2007; 1:89-99.

$146 \mathrm{Ma}$ L, Broomfield S, Lavery C, Lin SL, Xiao W, Bacchetti S. Up-regulation of CIR1/CROC1 expression upon cell immortalization and in tumor-derived human cell lines. Oncogene 1998; 17:1321-1326.

147 Andersen PL, Zhou H, Pastushok L, et al. Distinct regulation of Ubc13 functions by the two ubiquitin-conjugating enzyme variants Mms2 and Uev1A. J Cell Biol 2005; 170:745-755.

148 Lawrence CW, Hinkle DC. DNA polymerase $\zeta$ and the control of DNA damage induced mutagenesis in eukaryotes. Cancer Surv 1996; 28:21-31.

149 Tissier A, Kannouche P, Reck MP, Lehmann AR, Fuchs RP, Cordonnier A. Co-localization in replication foci and interaction of human Y-family members, DNA polymerase pol $\eta$ and REV1 protein. DNA Repair (Amst) 2004; 3:1503-1514.

150 O-Wang J, Kawamura K, Tada Y, et al. DNA polymerase $\kappa$, implicated in spontaneous and DNA damage-induced mutagenesis, is overexpressed in lung cancer. Cancer Res 2001; 61:5366-5369.

151 Pan Q, Fang Y, Xu Y, Zhang K, Hu X. Down-regulation of DNA polymerases $\kappa, \eta, 1$, and $\zeta$ in human lung, stomach, and colorectal cancers. Cancer Lett 2005; 217:139-147. 